\title{
An Inkjet Printed Meandered Dipole Antenna for RF Passive Sensing Applications
}

\author{
Abdul Quddious ${ }^{1}$, Munawar M. Khan ${ }^{1}$, Farooq A. Tahir ${ }^{1}$, A. Shamim², Hammad M. Cheema ${ }^{1}$ \\ ${ }^{1}$ School of Electrical Engineering \& Computer Science (SEECS), National University of Sciences \& \\ Technology (NUST), Islamabad, Pakistan \\ \{12mseeaquddious, 12mseemmkhan, farooq.tahir, hammad.cheema $\} @$ seecs.edu.pk \\ ${ }^{2}$ Department of Computer, Electrical and Mathematical Sciences and Engineering (CEMSE), King Abdullah \\ University of Sciences and Technology (KAUST), Thuwal, KSA, atif.shamim@kaust.edu.sa
}

\begin{abstract}
In this paper, a low cost inkjet printed antenna envisioned for integration with printed and non-printed RF sensors is presented. The proposed meandered dipole dual-loop antenna is designed on a $0.25 \mathrm{~mm}$ thick paper substrate. The antenna not only gives wireless remote sensing capability but also allows remote identification functionality. The antenna structure consists of an outer loop and an inner loop resonating at $3 \mathrm{GHz}$ and $5 \mathrm{GHz}$ respectively and used for obtaining unique electromagnetic signature by modifications in their dimensions.
\end{abstract}

Index Terms- Inkjet printing, paper substrate, dual-band antenna, wireless applications.

\section{INTRODUCTION}

In recent years there has been a lot of research and effort focused on combining wireless devices with sensors for collecting and processing of information about different objects, personnel and environment. For this purpose, RF identification has been successfully employed as a low cost, reduced size, reliable and lightweight solution, allowing passive wireless sensing capability. Some designs have been proposed in literature that use RFID technology for communicating sensor data, including temperature sensor [1], gas sensor [2] and humidity sensor [3]. Efforts have been made to reduce the cost of these sensors by using combination of discrete components and efficient inkjet printing techniques, to further simplify and at the same time enhance the capability of passive sensing through novel designs and systems [4-5].

In this work, a fully inkjet-printed communication module is presented for passive sensing applications. This module serves two purposes; one, it gives wireless capability to the sensor, and second, it allows unique identification. The space of $5.25 \mathrm{~cm}^{2}$ left intentionally in the center of the tag, it is used to integrate a fully printed sensor module in the form of interdigitated or metal-over-metal capacitors.

\section{ANTENNA DESIGN}

To give remote and wireless sensing functionality to an RF sensor, we have designed an antenna (shown in Fig. 1) that can be integrated with fully printed sensor, allowing the response of the sensor to be measured remotely. The proposed

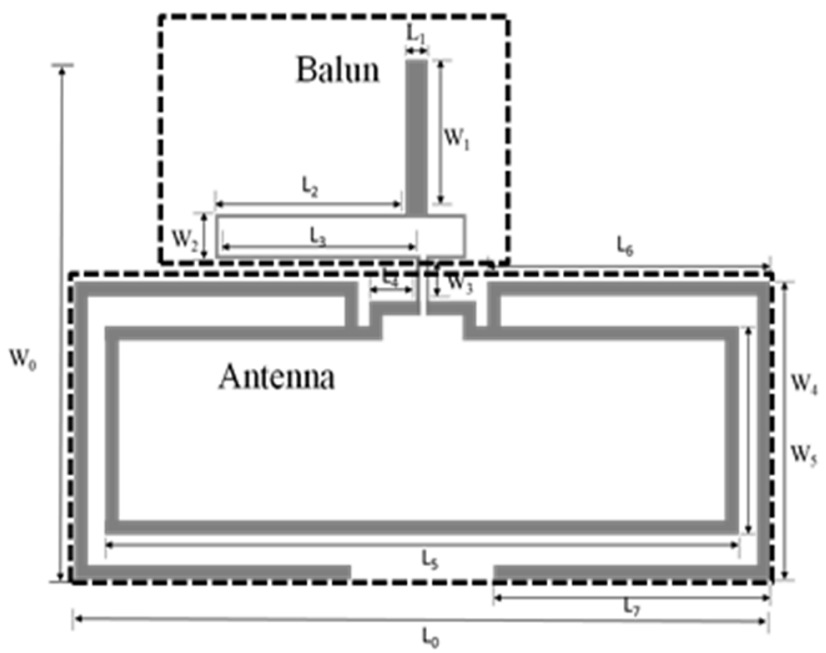

Fig. 1. Layout of the proposed meandered dipole loop antenna fed using balun. The wide space in the center is left on purpose for sensor integration.

meandered dipole dual-loop antenna (detailed dimensions in Table I) consists of two loops; the outer loop resonating at $3 \mathrm{GHz}$ and the inner loop resonating at $5 \mathrm{GHz}$. The band of operation for outer and inner loops is $2-3 \mathrm{GHz}$ and $5-6 \mathrm{GHz}$ respectively. The first band is used for tag identification and the second is used for sensor response.

TABLE I

DETAILED DIMENSIONS OF THE PROPOSED ANTENNA

\begin{tabular}{|c|c|c|c|}
\hline Parameter & Dimension & Parameter & Dimension \\
\hline $\mathrm{L}_{0}$ & $50.25 \mathrm{~mm}$ & $\mathrm{~W}_{0}$ & $35 \mathrm{~mm}$ \\
\hline $\mathrm{L}_{1}$ & $1.64 \mathrm{~mm}$ & $\mathrm{~W}_{1}$ & $10.75 \mathrm{~mm}$ \\
\hline $\mathrm{L}_{2}$ & $13.75 \mathrm{~mm}$ & $\mathrm{~W}_{2}$ & $3.0 \mathrm{~mm}$ \\
\hline $\mathrm{L}_{3}$ & $14.57 \mathrm{~mm}$ & $\mathrm{~W}_{3}$ & $2.75 \mathrm{~mm}$ \\
\hline $\mathrm{L}_{4}$ & $3.5 \mathrm{~mm}$ & $\mathrm{~W}_{4}$ & $14 \mathrm{~mm}$ \\
\hline $\mathrm{L}_{5}$ & $45.75 \mathrm{~mm}$ & $\mathrm{~W}_{5}$ & $20 \mathrm{~mm}$ \\
\hline $\mathrm{L}_{6}$ & $20.5 \mathrm{~mm}$ & $\mathrm{~L}_{7}$ & $20 \mathrm{~mm}$ \\
\hline
\end{tabular}




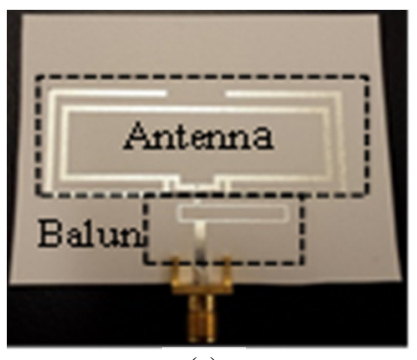

(a)

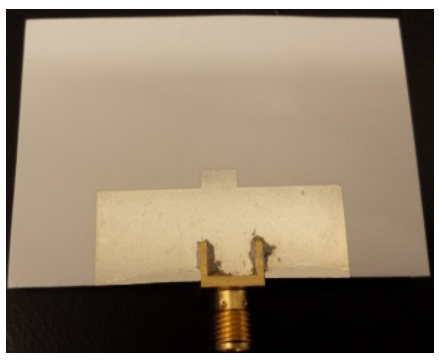

(b)
Fig. 2. Fabricated prototypes (a) Top view of antenna (b) Bottom view

For the antenna, the resonance frequency and bandwidth are set by varying the geometric parameter such as; horizontal stub lengths $\mathrm{L}_{5}, \mathrm{~L}_{6}, \mathrm{~L}_{7}$, and vertical stub lengths $\mathrm{W}_{4}$ and $\mathrm{W}_{5}$. A parametric sweep is performed in simulations, and the final optimized parameters of the antenna are shown in Table I. The horizontal stub lengths $\mathrm{L}_{6}$ and $\mathrm{L}_{7}$ control the resonance frequency in the identification band. Increasing $\mathrm{L}_{6}$ and $\mathrm{L}_{7}$ shifts the resonating frequency to a lower band while maintaining the operational bandwidth due to constant vertical stub length.

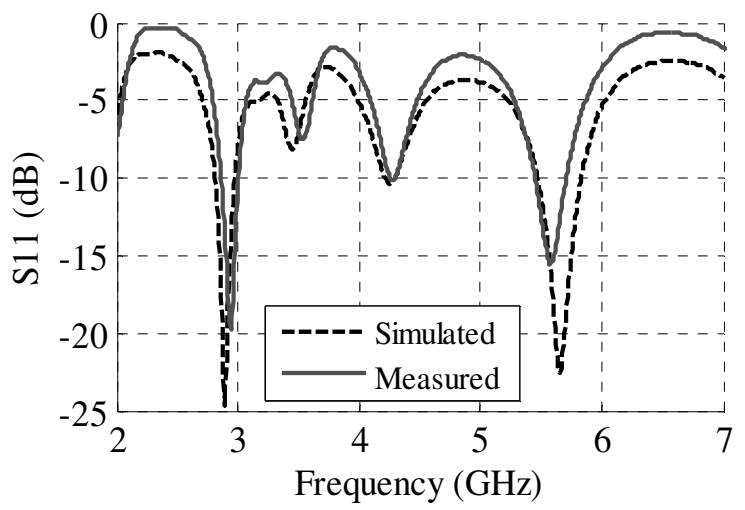

Fig. 3. Simulated and measured return loss of antenna on Teslin paper.

In order to measure the dipole antenna via coaxial cable, a balance-to-unbalance transformer (balun) is used. Many approaches for balun realizations are reported in the literature [6]. In this paper, the balun is realized by printing it on the same layer as that of the antenna. The partial ground plane on the back side acts as a ground reference for the balun. The tag is made using two layers of paper substrate: antenna with balun is printed on one layer while the partial ground plane is realized on the second layer. The two layers are then glued together. The balun introduces 180 degree phase delay between the two microstrip lines of the dipole of $50 \Omega$ impedance. To obtain the required phase delay, the difference in the length at the center frequency of the two branches is approximately $\frac{\lambda}{2}$.

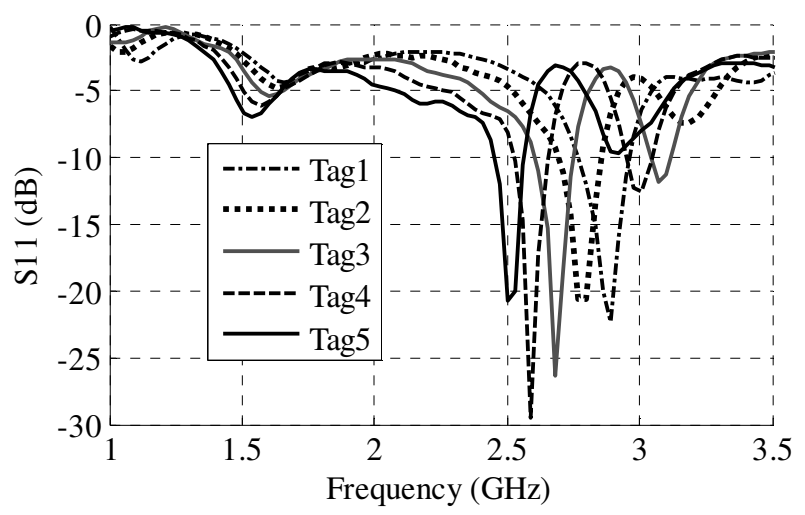

Fig. 4. Antenna response used for identification.

\section{FABRICATION AND RESULTS}

The antenna is designed and simulated using CST Microwave Studio. The fabricated prototype of the antenna is shown in Fig. 2. The antenna is fabricated via inkjet printing using Dimatix DMP-2831 printer and UT Dots silver nanoparticle ink. Teslin paper substrate is used with permittivity 2 , loss tangent 0.02 and thickness of $0.25 \mathrm{~mm}$. To obtain accurate dimensions as well as good conductivity, the nanoparticle drop spacing and number of layers are carefully chosen. An optimized drop spacing of $35 \mu \mathrm{m}$ and 5 conductive layers on top of each other are used. After printing, the antenna is heat sintered at $120^{\circ} \mathrm{C}$ for 10 minutes. The conductivity thus achieved is approximately $6 \mathrm{e}^{6} \mathrm{~S} / \mathrm{m}$. An SMA connector is mounted using conductive silver epoxy. The prototypes are characterized in terms of return loss using an Agilent vector network analyzer.

The simulated and measured results are in good agreement with each other as shown in Fig. 3. The graph shows two distinct bands at $2.8 \mathrm{GHz}$ and $5.6 \mathrm{GHz}$. The $2.8 \mathrm{GHz}$ band response is due to the outer loop of the antenna while $5.6 \mathrm{GHz}$ response is due to inner loop. By changing the dimensions $\mathrm{L}_{6}$ and $\mathrm{L}_{7}$, a frequency shift in the lower band is achieved. The response is shown in Fig. 4, whereas dimensions are shown in Table II. This shift in the frequency is unique for each tag and is used for its identification. The $5.6 \mathrm{GHz}$ band can be used for remote sensing, which would change/shift in frequency as response of the RF sensor changes.

TABLE II CHANGE IN DIMENSIONS FOR UNIQUE IDENTIFICATION

\begin{tabular}{|c|c|c|}
\cline { 2 - 3 } \multicolumn{1}{c|}{} & $\mathrm{L}_{6}$ & $\mathrm{~L}_{7}$ \\
\hline Tag 1 & $20.5 \mathrm{~mm}$ & $20 \mathrm{~mm}$ \\
\hline Tag 2 & $21.5 \mathrm{~mm}$ & $21 \mathrm{~mm}$ \\
\hline Tag 3 & $22.5 \mathrm{~mm}$ & $22 \mathrm{~mm}$ \\
\hline Tag 4 & $23.5 \mathrm{~mm}$ & $23 \mathrm{~mm}$ \\
\hline Tag 5 & $24.5 \mathrm{~mm}$ & $24 \mathrm{~mm}$ \\
\hline
\end{tabular}




\section{CONCLUSION}

Research and development towards the realization of fully printed passive sensor tags has increased in the recent years. It involves two tasks; realization of a fully printed antenna along with a fully printed sensor module, and their integration. In this paper, a wireless sensor-identifying module that serves the purpose of identification and communicating sensed data is designed and implemented. This module consists of a meandered dipole dual-loop antenna that is fully printed without the use of any discrete components. A wide space of $5.25 \mathrm{~cm}^{2}$ is left in the center that serves the purpose of future integration with sensing module.

\section{REFERENCES}

[1] J. Gao, J. Sidn, and H. E. Nilsson, "Printed temperature sensors for passive RFID tags," presented at the Progress In Electromagnetics Research Symp., Xi'an, China, 2010.

[2] L. Yang, R. Zhang, D. Staiculescu, C. Wong, and M. Tentzeris, "A novel conformal RFID-enabled module utilizing inkjet-printed antennas and carbon nanotubes for gas-detection applications," IEEE Antennas Wireless Propag. Lett. vol. 8, pp. 653-656, 2009.

[3] J. Gao, J. Sidén, H-E Nilsson, and M. Gulliksson, "Printed Humidity Sensor With Memory Functionality for Passive RFID Tags," IEEE Sens J, Vol. 13, No. 5, 2013.

[4] Munawar M. Khan, Farooq A. Tahir, M. F. Farooqui, Atif Shamim, Hammad M. Cheema, "3.56 bits/ $/ \mathrm{cm}^{2}$ Compact Inkjet Printed and Application Specific Chipless RFID Tag", IEEE Antenna and Wireless Propagation Letters, 2015.

[5] Sana Ahmed, Farooq A. Tahir, A. Shamim, Hammad M. Cheema, "A Compact Kapton-based Inkjet Printed Multiband Antenna for Flexible Wireless Devices", IEEE Antenna and Wireless Propagation Letters, vol. 14, pp. 1802-1805, 2015.

[6] R. Cosmina Hadarig, M. E. de Cos, and F. Las-Heras, "UHF dipoleAMC combination for RFID applications," IEEE Antennas Wirel. Propag. Lett. vol. 12, pp. 1041-1044, 2013. 